\title{
WALKING THE TALK? Jesuit Universities and Fossil Fuel Investments
}

\author{
MARINILKA BARROS KIMBRO (corresponding author) \\ Albers School of Business \& Economics \\ Seattle University, Seattle, Washington, U.S.A \\ kimbrom@seattleu.edu
}

RUBINA MAHSUD

Albers School of Business \& Economics

Seattle University, Seattle, Washington, U.S.A

mahsudr@seattleu.edu

DAVIT ADUT

Albers School of Business \& Economics

Seattle University, Seattle, Washington, U.S.A

adutd@seattleu.edu

\section{ABSTRACT}

The student-led fossil fuel divestment (FFD) movement urges universities to remove investments in fossil fuel firms from their endowment portfolios to inspire reductions in carbon emissions and help control climate change. This article explores the movement in U.S.-based Jesuit universities by documenting their endowment size, current divestment status, and rationale for or against divesting. These institutions held a total of US $\$ 13.8$ billion in their endowments as of 2019, making their investment decisions relevant and material.

The article in general examines the alignment of divestment actions with the commitments of Jesuit universities to environmental stewardship and social justice as expressed in their mission statements and Pope Francis's encyclical Laudato Si'. Two out of the 27 Jesuit universities in the U.S., namely, Georgetown and Seattle University, were already committed to FFD by April 2020; after accounting for branch campuses, this represents a commitment of $13.3 \%$ among all U.S.-based Jesuit universities. This is appreciably higher than the $4.12 \%$ divestment rate among all private 4-year universities in the United States.

Each of the 27 U.S.-based Jesuit universities was contacted to verify their endowment size, divestment status, and position on FFD. The 13 who responded stated their commitment to environmental protection and sustainability, and some reported their rationale for or against divesting. Results suggest that the investment strategies of Jesuit universities are a "work in progress," and are likely to evolve as they align with their common Roman Catholic and Jesuit identity and mission. 
The reasons stated for deciding not to divest, moreover, are consistent with previous literature. A second article in this issue of the Journal of Management for Global Sustainability explores those reasons in detail and broadens the theme of divesting to encompass any organization.

\title{
KEYWORDS
}

investments of Jesuit universities; divestment from fossil fuel firms;

fossil fuel-free endowments; socially responsible investments in higher education; environmental justice and ethics; Laudato $\mathrm{Si}^{\prime}$

\section{INTRODUCTION}

\begin{abstract}
A very solid scientific consensus indicates that we are presently witnessing a disturbing warming of the climatic system ... [and] a number of scientific studies indicate that most global warming in recent decades is due to the great concentration of greenhouse gases (carbon dioxide, methane, nitrogen oxides and others) released mainly as a result of human activity.
\end{abstract}

If present trends continue, this century may well witness extraordinary climate change and an unprecedented destruction of ecosystems, with serious consequences for all of us.

Laudato $\mathrm{Si}^{\prime}$ is probably one of the most eloquent attempts to articulate the moral imperative of addressing climate change. A chemist by training, Francis compellingly affirms that global warming, caused primarily by greenhouse gases generated from the burning of fossil fuels, is a human-made problem. One of his central themes echoes Bartholomew, the "Green Patriarch" of Orthodox Christians who calls on all human beings to repent for the ecological damage they have caused (Rich, 2018).

Responding, in part, to Laudato $\mathrm{Si}^{\prime}$, students in various Jesuit universities have joined the "fossil free" movement, organizing efficiently and collaborating through collective exchanges such as the Jesuit Divestment Network. Ever since the emergence of the fossil fuel divestment (FFD) movement in the U.S. in the last decade, university students have been urging their institutions to remove investments in fossil fuel firms from their endowment portfolios, all in an attempt to inspire social, political, and 
economic actions that would ultimately reduce carbon emissions and control climate change (Linnenluecke, Meath, Rekker, Sidhu, \& Smith, 2015). By April of 2020, two out of the 27 Jesuit universities based in the U.S. had agreed to divest, or "dis-invest" from or sell, any and all financial holdings in fossil fuel firms. After accounting for branch campuses, this means that $13.33 \%$ of all Jesuit higher education institutions in the U.S. have adopted an FFD strategy; in comparison, only $4.12 \%$ of all 4 -year private institutions in the U.S. (counting all branches) have agreed to divest from fossil fuels. These results are encouraging as they reflect a strong commitment to FFD and to environmental sustainability in general among Jesuit universities. This study, therefore, investigates if and how the investment decisions of Jesuit universities do reflect the message about fossil fuel impacts on climate change as outlined in Laudato $\mathrm{Si}^{\prime}$ and elsewhere.

Data on FFD was obtained from the 350.org public database while endowment data was acquired through the National Association of College and University Business Officers (NACUBO). The websites of all 27 U.S.-based Jesuit universities were also examined to investigate their 1) general environmental/sustainability initiatives; 2) divestment status, and 3) endowment investment policy (if available). All these institutions were contacted to verify and for comment on collected endowment and divestment status data as well as to request for information about their policies regarding investments in fossil fuel firms. ${ }^{1}$

Of the 27 Jesuit universities that were contacted, 13 responded (48.15\%), with ten of those providing, in addition, various degrees of information regarding their environmental initiatives as well as rationale for and against divesting from fossil fuel firms. The results indicate that even though all U.S.-based Jesuit universities advocate explicitly for environmental stewardship and for the creation of a sustainable world in various forms, the majority are still invested in the stocks of firms that are among the 200 largest in the world in terms of oil, gas, and carbon reserves (see Table 1). Only two U.S.-based Jesuit universities so far-Georgetown University and Seattle University-have affirmatively committed to a fossil fuel-free investment portfolio.

In the case of non-divesting Jesuit universities in the U.S., respondents alluded to various reasons for rejecting an FFD strategy, all of which were linked to financial

${ }^{1}$ A list of which companies are considered "fossil fuel firms" was also sent to all these universities. See Table 1. 
concerns or, rather, a cost-benefit logic. They stated, for example, that they do not have direct control of investments, or that they rely on the investments of index/ mutual funds that include stocks in fossil fuel firms. Non-divesting universities argued that divesting creates high transaction costs, increases portfolio risk, reduces investment income, and impairs diversification benefits, that it is a "political issue," will not make a difference in carbon emissions, and is contradictory to their own use of fossil fuels in other activities (all these arguments against divesting are discussed in detail in the next article of this issue of the Journal).

The remainder of this article is structured as follows. The second section provides an overview of the role played by fossil fuel firms' carbon emissions in global warming, along with some strategies for dealing with, as well as responses to, the current climate crisis. The third section offers a brief history of divestment movements that leads to the current FFD initiative in higher education. The fourth section describes the methodology used to explore this topic and the data developed from it. Lastly, the fifth section points readers toward the second article in this issue of the Journal which examines and discusses the alleged reasons for maintaining endowment portfolios that carry investments in fossil fuel securities, and offers some possible next steps following that analysis.

This first article, then, can be viewed as asking the "what's so?" question about climate change, as well as "so what?" concerning university investments in fossil fuel firms. The second article, on the other hand, can be viewed as starting to address a "now what?" question arising from attempts to answer the "what's so?" and "so what?" concerns explored in this article: what might universities do with their investments to deal with the threats of climate change and global unsustainability?

\section{BACKGROUND}

\section{Fossil Fuels and Climate Change}

On June 23, 1988, the director of NASA's Goddard Institute for Space Studies, James Hansen, delivered historic testimony to the U.S. Congress (Shabecoff, 1988). He declared science to be $99 \%$ unequivocal that the world was warming and that humans, by burning fossil fuels and through other activities, had altered the global climate in a manner that was going to change life dramatically on Earth. In that 
testimony, he predicted (almost a decade earlier) that there could be an increase of two degrees Centigrade in global temperature if these trends continued, which would result in massive droughts, floods, thermal expansion of the oceans, the melting of glaciers, the rise of sea levels by as much as one to four feet, and the destruction of coastal cities, reefs, and most living species by 2050.

Indeed, the Earth's temperature has since risen by an average of one degree Centigrade ${ }^{2}$ from pre-industrial levels (1850-1900), and the planet has experienced an astonishing run of record-breaking heat waves, hurricanes, storms, forest fires, droughts, and floods, as well as rising sea levels and ocean acidification caused by carbon dioxide ( $\mathrm{CO}_{2}$ ) emissions (see Gillis, 2018). February 2020 was 1.17 degrees Centigrade warmer than the recorded average, and according to the National Oceanic and Atmospheric Administration (NOAA), there is a $75 \%$ probability that 2020 will set a record for the warmest year to date (Freedman, 2020). The United Nations' Intergovernmental Panel on Climate Change Report for Policymakers (IPCC, 2018), moreover, concluded in October of 2018 that the average global temperature will probably rise by 1.5 degrees Centigrade $^{3}$ by 2030 - and almost certainly by 2040 - if no major cuts in $\mathrm{CO}_{2}$ emissions occur. In fact, the report stated that even if such cuts were to begin immediately, they would only delay and not prevent this increase (Goodwin, Katavouta, Roussenov, Foster, Rohling, \& Williams, 2018).

Drawing upon the work of scores of scientists, Elizabeth Kolbert also predicts that human activity and climate change will probably cause the extinction of $20 \%$ to $50 \%$ of all living species by the end of this century in what she calls the "sixth great extinction" (Kolbert, 2014). Indeed, the World Wildlife Fund (WWF), echoing Kolbert in its Living Planet Report, reports overall declines of $60 \%$ and $83 \%$ in vertebrate and freshwater species, respectively, between 1970 and 2014 (WWF, 2018). Unfortunately, the precipitous decline in earth's biodiversity and the destruction of animal and plant species can be traced back convincingly to human activity, for which deforestation, global warming, and pollution are significant drivers. The Earth, as Kolbert warns, is in the midst of a modern and anthropogenic sixth extinction that will likely be humanity's most lasting 
legacy, thereby challenging us to rethink the fundamental question of what it means to be human.

\section{Individual and Collective Responses to the Climate Crisis}

In addition to using our voices to communicate that human beings have altered the environment and caused climate change, there are objective and measurable actions that we all can take on a personal level to reduce pollution and waste (Hawken, 2017; Weis, 2013). There is consensus, however, that changes by individuals alone cannot prevent environmental disaster. One perspective that focuses on the national level, for instance, observes that only three countries are responsible for more than a third of all greenhouse gases emitted worldwide. According to the European Commission's 2018 Fossil $\mathrm{CO}_{2}$ Emissions of All World Countries report (Muntean et al., 2018), the United States contributes 13.8\% while China's and India's carbon footprints continue to increase, standing at $29.3 \%$ and $6.6 \%$, respectively, at the time of the report. ${ }^{4}$ Yet while a nation-based approach to assigning responsibility for carbon emissions is useful for the design and formation of multinational cooperation agreements within the jurisdiction of international law, action at the political level has proven to be disappointing while regulatory efforts seem perpetually stymied by powerful private interests. Global treaties, in addition, remain entangled in governmental disagreements over who should bear the cost.

Another strategy is based on the idea of tracing emissions-and responsibilityto the direct producers of greenhouse gases. Heede (2014), for instance, quantified the fossil fuel production records of firms from 1854 to 2010 and concluded that $63 \%$ of all worldwide emissions can be attributed to only 90 companies. His results offer the opportunity to assign responsibility for causing — and remedying — climate change to those firms who own and market fossil fuels.

Linnenluecke et al. (2015), on the other hand, argue that a developing confluence between policy and organizational responses has the potential to lead to greater action on climate change (Gunningham, 2017). McKibben (2013) proposes a related approach: a collective campaign on divestment which, he argues, will shift public opinion, stigmatize the fossil fuel industry, and mobilize pressure on community

${ }^{4} \mathrm{Among}$ these three countries, the United States is the largest contributor of greenhouse gases $(\mathrm{GHG}$ ) on a per capita basis (in tons): $U S=15.7$; China $=7.7$; and India $=1.8$. 
leaders and politicians to address what McKibben described as "the greatest intellectual and moral problem in human history" (emphasis added).

\section{DIVESTMENT: A STRATEGY FOR SOCIAL JUSTICE}

Led by the Reverend Leon Sullivan and several religious institutions, the late $20^{\text {th }}$-century movement against the South African apartheid regime was the first major divestment campaign in modern history. It urged all U.S. firms to stop doing business in South Africa, where apartheid laws once required all companies operating within South African borders to follow rules that had been explicitly designed to protect white supremacy (Seidman, 2015; Apfel, 2015). The pressure for divestment grew in intensity over time, and firms were eventually pressured to withdraw from the country (Hiltzik, 2016). Indeed, after apartheid was dismantled in the early 1990s, ethical investing initiatives began targeting the arms trade, tobacco firms, heavy polluters, and human rights violators (Loder, 2017).

The history of divestment in the Roman Catholic Church and among other religious organizations in general has been well documented (Krantz, 2015; Hodgson, 2009; Clark, 2011; Roewe, 2014, 2016; Finn, 2019). Roman Catholic institutions in the early twentieth century expressed their faith and values in their investments and "screened" alcohol and tobacco firms from their portfolios. Fifty years later, Christian/Catholic funds began screening for weapons manufacturers. This process, in general, was undertaken not with the hope of having a major financial impact on these companies but rather to align investors' values and beliefs with their assets as well as for any symbolic impact such actions might have on society.

Divestment campaigns, to some extent, attempt to fill the void left by political and governmental inaction. When governments seem unable or unwilling to assert a larger public interest in dealing with corporations, and where private gain seems to come at the expense of a larger public good, divestment discussions provide an avenue for garnering attention across an array of local communities, pushing leaders to respond when private behaviors contribute to, or profit from, a global problem. In calling attention to large issues that link organizational practices to broader social problems, divestment movements seek to spark a sense of moral urgency and create support for strong intervention. By encouraging individuals to focus on institutional relationships, they can prompt 
examinations of important global problems and offer concrete means for individuals and organizations to demonstrate explicitly, through collective action, what they believe in and care for (Benford, 1993; Kimbro, 2018).

An example of an attempt to fill the void left by a lack of appropriate government action was the response of Dick's Sporting Goods after the Parkland High School massacre in Florida in February of 2018 (Barca, 2018). In response to this tragedy, the company decided to stop selling assault rifles and high-capacity magazines. It is worth noting, moreover, as in the case of the anti-apartheid movement, that a religious institution was actively involved in encouraging Dick's to stop selling those products-the Sisters of Mercy had been lobbying the company ever since to restrict its firearms merchandise. Thus, when the Parkland shooting happened, Dick's took the step that the Sisters and their coalition allies had been advocating for and made the policy shift.

The dilemma, on the other hand, of whether firms (or institutions) have a fiduciary duty to shareholders (Friedman, 1970) or to society was and continues to be a heavily debated topic (Barnett, 2007). Some business leaders, following Milton Friedman's view in his widely-cited 1970 essay in the New York Times Magazine, still insist that their only ethical obligation is to focus on profit maximization within the laws and regulatory structure of the countries in which they operate.

\section{Fossil Fuel Divestment Movement}

FFD campaigns urge investors to sell their stakes in companies that hold reserves of and supply coal, oil, or gas. Those who do so would be taking a step toward aligning ethical concerns with investment decisions, for the moral and practical framing of FFD is predicated on the argument that investing in fossil fuel firms ultimately legitimizes their past, present, and future activities. It maintains that the business model of the fossil fuel industry is unsustainable and will ultimately lead to an uninhabitable planet. Indeed, one of the movement's goals is to help people realize that the role played by fossil fuel companies in society is like the role played by tobacco firms in one's health-as hazards to life. Owning stock in a fossil fuel firm, in other words, affirmatively legitimizes the destruction that climate change has caused, is causing, and will cause. 
With its aim of building support for legislation and technology that reduce greenhouse gas emissions by cutting down financial support for fossil fuel investments and operations while addressing the moral legitimacy of fossil fuel production and its use (Ansar, Caldecott, \& Tilbury, 2013; Ayling \& Gunningham, 2017), FFD has gained traction among foundations, pension funds, faith-based organizations, governments, and other organizations all over the world. As of April 2020, \$14.14 trillion worth of assets under management (AuM) have been committed to fossil fuel divestment ${ }^{5}$ (Fossil Free, n.d.) while FFD pledges to divest $\$ 5.2$ billion have been made by 1,195 institutions and 58,000 individuals. Educational institutions represent 15\% of all divestments while faith-based institutions, interestingly enough, comprise the largest group, representing $30 \%$ of all divestment commitments.

One of the movement's largest victories was when Norway's \$900 billion sovereign-wealth fund agreed in June of 2015 to sell $\$ 9$ billion worth of stocks in firms that mine coal and tar sands. Success continued in 2018, when New York City announced that it would fully divest fossil fuel companies from its roughly $\$ 5$ billion worth of pension funds within the next five years (Neuman, 2018).

It is necessary, of course, to determine which firms should be divested from if an FFD strategy is to be pursued. One approach is to select firms based on the fossil fuel reserves under their ownership and/or control. Developed by The Carbon Underground, Table 1 lists the top 100 firms according to coal reserves and the top 100 according to oil and gas (Fossil Free Indexes, 2018). These numbers represent the potential impact on present and future generations if these reserves are burned.

\begin{tabular}{|c|c|c|c|c|c|c|c|c|c|}
\hline \multicolumn{4}{|c|}{$\begin{array}{l}\text { COAL RESERVES } \\
\text { (Top } 100 \text { Firms) }\end{array}$} & \multicolumn{6}{|c|}{$\begin{array}{l}\text { OIL \& GAS RESERVES } \\
\text { (Top } 100 \text { Firms) }\end{array}$} \\
\hline & \multirow{2}{*}{ Firms } & \multirow{2}{*}{ Ticker } & \multirow{2}{*}{ Gt $\mathrm{CO}_{2}$} & & \multirow{2}{*}{ Firms } & \multirow{2}{*}{ Ticker } & Oil & Gas & O\&G \\
\hline & & & & & & & Gt $\mathrm{CO}_{2}$ & Gt $\mathrm{CO}_{2}$ & Gt $\mathrm{CO}_{2}$ \\
\hline 1 & $\begin{array}{l}\text { Coal } \\
\text { India }\end{array}$ & COALINDIA & 33.272 & 1 & Gazprom & GAZP & 35.116 & 4.345 & 39.462 \\
\hline 2 & $\begin{array}{c}\text { Adani } \\
\text { Enterprises }\end{array}$ & ADANIENT & 27.321 & 2 & Rosneft & ROSN & 3.413 & 11.574 & 14.987 \\
\hline 3 & $\begin{array}{l}\text { Shaanxi } \\
\text { Coal } \\
\text { Industry }\end{array}$ & 601225 & 27.152 & 3 & PetroChina & 0857 & 4.211 & 3.335 & 7.545 \\
\hline
\end{tabular}

${ }^{5}$ This includes full and partial commitments and divestments from coal and tar sands. 


\begin{tabular}{|c|c|c|c|c|c|c|c|c|c|}
\hline \multicolumn{4}{|c|}{$\begin{array}{l}\text { COAL RESERVES } \\
\text { (Top } 100 \text { Firms) }\end{array}$} & \multicolumn{6}{|c|}{$\begin{array}{l}\text { OIL \& GAS RESERVES } \\
\text { (Top } 100 \text { Firms) }\end{array}$} \\
\hline & \multirow{2}{*}{ Firms } & \multirow{2}{*}{ Ticker } & \multirow{2}{*}{ Gt $\mathrm{CO}_{2}$} & & \multirow{2}{*}{ Firms } & \multirow{2}{*}{ Ticker } & Oil & Gas & $O \& G$ \\
\hline & & & & & & & Gt CO & $\mathrm{Gt} \mathrm{CO}{ }_{2}$ & Gt $\mathrm{CO}_{2}$ \\
\hline 4 & $\begin{array}{c}\text { China } \\
\text { Shenhua } \\
\text { Energy }\end{array}$ & 1088 & 20.892 & 4 & $\begin{array}{l}\text { Exxon } \\
\text { Mobil }\end{array}$ & XOM & 3.006 & 4.487 & 7.492 \\
\hline 5 & Glencore & GLEN & 17.993 & 5 & $\mathrm{BP}$ & $\mathrm{BP}$ & 2.456 & 4.452 & 6.908 \\
\hline 6 & $\begin{array}{c}\text { BHP } \\
\text { Billiton }\end{array}$ & BHP & 12.707 & 6 & Lukoil & $\mathrm{LKOH}$ & 1.289 & 5.113 & 6.402 \\
\hline 7 & $\begin{array}{c}\text { Yanzhou } \\
\text { Coal } \\
\text { Mining }\end{array}$ & 1171 & 10.610 & 7 & Novatek & NVTK & 4.038 & 0.593 & 4.630 \\
\hline 8 & $\begin{array}{c}\text { China } \\
\text { Coal } \\
\text { Energy }\end{array}$ & 1898 & 9.875 & 8 & $\begin{array}{c}\text { Royal } \\
\text { Dutch } \\
\text { Shell }\end{array}$ & RDSA & 2.204 & 2.055 & 4.258 \\
\hline 9 & $\begin{array}{c}\text { Exxaro } \\
\text { Resources }\end{array}$ & $\mathrm{EXX}$ & 9.760 & 9 & Chevron & CVX & 1.675 & 2.583 & 4.258 \\
\hline 10 & $\begin{array}{l}\text { Public } \\
\text { Power }\end{array}$ & PPC & 9.339 & 10 & Petrobras & PBR & 0.431 & 3.571 & 4.002 \\
\hline 11 & Mitsubishi & 8058 & 8.902 & 11 & $\begin{array}{c}\text { Gazprom } \\
\text { Neft }\end{array}$ & SIBN & 1.186 & 2.665 & 3.851 \\
\hline 12 & $\begin{array}{l}\text { Inner } \\
\text { Mongolia } \\
\text { Yitai Coal }\end{array}$ & 3948 & 8.415 & 12 & Total & $\mathrm{FP}$ & 1.772 & 2.060 & 3.832 \\
\hline 13 & $\begin{array}{c}\text { Peabody } \\
\text { Energy }\end{array}$ & BTU & 7.767 & 13 & ENI & ENI & 1.061 & 1.449 & 2.745 \\
\hline 14 & $\begin{array}{c}\text { Yancoal } \\
\text { Australia }\end{array}$ & YAL & 6.945 & 14 & Tatneft & TATN & 0.000 & 2.631 & 2.631 \\
\hline 15 & $\begin{array}{c}\text { Foresight } \\
\text { Energy }\end{array}$ & FELP & 6.759 & 15 & ONGC & ONGC & 0.822 & 1.690 & 2.512 \\
\hline 16 & $\begin{array}{c}\text { Shanxi } \\
\text { Xishan } \\
\text { Coal \& } \\
\text { Elect }\end{array}$ & 000983 & 5.416 & 16 & Statoil & EQNR & 0.821 & 1.135 & 1.957 \\
\hline 17 & Mechel & MTLR & 5.203 & 17 & $\begin{array}{c}\text { Cono- } \\
\text { coPhillips }\end{array}$ & COP & 0.649 & 1.160 & 1.809 \\
\hline 18 & $\begin{array}{c}\text { Anglo } \\
\text { American }\end{array}$ & AAL & 5.145 & 18 & CNOOC & 0883 & 0.450 & 1.217 & 1.667 \\
\hline 19 & $\begin{array}{c}\text { BUMI } \\
\text { Resources }\end{array}$ & BUMI & 5.103 & 19 & $\begin{array}{c}\text { Canadian } \\
\text { Nat } \\
\text { Resources } \\
\end{array}$ & CNQ & 0.331 & 1.271 & 1.602 \\
\hline 20 & $\begin{array}{c}\text { Whitehaven } \\
\text { Coal }\end{array}$ & WHC & 5.067 & 20 & Inpex & 1605 & 0.321 & 0.936 & 1.257 \\
\hline
\end{tabular}




\begin{tabular}{|c|c|c|c|c|c|c|c|c|c|}
\hline \multicolumn{4}{|c|}{$\begin{array}{l}\text { COAL RESERVES } \\
\text { (Top } 100 \text { Firms) }\end{array}$} & \multicolumn{6}{|c|}{$\begin{array}{l}\text { OIL \& GAS RESERVES } \\
\text { (Top } 100 \text { Firms) }\end{array}$} \\
\hline & \multirow{2}{*}{ Firms } & \multirow{2}{*}{ Ticker } & \multirow{2}{*}{ Gt CO $\mathrm{CO}_{2}$} & & \multirow{2}{*}{ Firms } & \multirow{2}{*}{ Ticker } & Oil & Gas & O\&G \\
\hline & & & & & & & $\mathrm{GtCO}_{2}$ & $\mathrm{Gt} \mathrm{CO}_{2}$ & $\mathrm{Gt} \mathrm{CO}_{2}$ \\
\hline 21 & $\begin{array}{c}\text { China } \\
\text { Coal } \\
\text { Xinji } \\
\text { Energy }\end{array}$ & 601918 & 5.056 & 21 & EQT & EQT & 1.081 & 0.076 & 1.156 \\
\hline 22 & $\begin{array}{c}\text { Tambang } \\
\text { Batubara } \\
\text { Bukit } \\
\text { Asam } \\
\text { Aktie }\end{array}$ & PTBA & 5.052 & 22 & Sinopec & SINOPEC & 0.381 & 0.677 & 1.058 \\
\hline 23 & $\begin{array}{l}\text { Lu'an } \\
\text { Env } \\
\text { Energy }\end{array}$ & 601699 & 4.748 & 23 & Bashneft & BANE & 0.000 & 1.036 & 1.036 \\
\hline 24 & Vale & VALE3 & 4.162 & 24 & Repsol & REP & 0.681 & 0.322 & 1.003 \\
\hline 25 & $\begin{array}{l}\text { Kailuan } \\
\text { Energy } \\
\text { Chemical }\end{array}$ & 600997 & 4.155 & 25 & Occidental & OXY & 0.209 & 0.776 & 0.984 \\
\hline 26 & $\begin{array}{c}\text { Teck } \\
\text { Resources }\end{array}$ & TCK.B & 4.124 & 26 & $\begin{array}{c}\text { EOG } \\
\text { Resources }\end{array}$ & EOG & 0.232 & 0.712 & 0.945 \\
\hline 27 & $\begin{array}{l}\text { Arch } \\
\text { Coal }\end{array}$ & $\mathrm{ARCH}$ & 3.838 & 27 & $\begin{array}{c}\text { Antero } \\
\text { Resources }\end{array}$ & $\mathrm{AR}$ & 0.605 & 0.290 & 0.895 \\
\hline 28 & $\begin{array}{l}\text { Alliance } \\
\text { Resource } \\
\text { Partners }\end{array}$ & ARLP & 3.626 & 28 & $\begin{array}{c}\text { Range } \\
\text { Resources }\end{array}$ & RRC & 0.559 & 0.242 & 0.802 \\
\hline 29 & $\begin{array}{c}\text { Raspadskaya } \\
\text { OAO }\end{array}$ & RASP & 3.616 & 29 & $\begin{array}{l}\text { South- } \\
\text { western } \\
\text { Energy }\end{array}$ & SWN & 0.606 & 0.179 & 0.785 \\
\hline 30 & $\begin{array}{c}\text { DaTong } \\
\text { Coal } \\
\text { Industry }\end{array}$ & 601001 & 3.508 & 30 & $\begin{array}{l}\text { Suncor } \\
\text { Energy }\end{array}$ & SU & 0.001 & 0.708 & 0.709 \\
\hline 31 & EVRAZ & EVR & 2.997 & 31 & $\begin{array}{c}\text { Cenovus } \\
\text { Energy }\end{array}$ & CVE & 0.107 & 0.600 & 0.707 \\
\hline 32 & Sasol & SOL & 2.897 & 32 & $\begin{array}{l}\text { Noble } \\
\text { Energy }\end{array}$ & NBL & 0.420 & 0.268 & 0.689 \\
\hline 33 & $\begin{array}{c}\text { Beijing } \\
\text { Haohua } \\
\text { Energy } \\
\text { Res }\end{array}$ & 601101 & 2.676 & 33 & $\begin{array}{l}\text { Devon } \\
\text { Energy }\end{array}$ & DVN & 0.326 & 0.332 & 0.658 \\
\hline 34 & $\begin{array}{l}\text { Westmo- } \\
\text { reland } \\
\text { Coal }\end{array}$ & WLB & 2.649 & 34 & $\begin{array}{c}\text { Chesapeake } \\
\text { Energy }\end{array}$ & $\mathrm{CHK}$ & 0.469 & 0.175 & 0.643 \\
\hline
\end{tabular}




\begin{tabular}{|c|c|c|c|c|c|c|c|c|c|}
\hline \multicolumn{4}{|c|}{$\begin{array}{l}\text { COAL RESERVES } \\
\text { (Top } 100 \text { Firms) }\end{array}$} & \multicolumn{6}{|c|}{$\begin{array}{c}\text { OIL \& GAS RESERVES } \\
\text { (Top } 100 \text { Firms) }\end{array}$} \\
\hline & \multirow{2}{*}{ Firms } & \multirow{2}{*}{ Ticker } & \multirow{2}{*}{ Gt $\mathrm{CO}_{2}$} & & \multirow{2}{*}{ Firms } & \multirow{2}{*}{ Ticker } & Oil & Gas & O\&G \\
\hline & & & & & & & Gt CO & $\mathrm{GtCO}_{2}$ & $\mathrm{Gt} \mathrm{CO}$ \\
\hline 35 & ITOCHU & 8001 & 2.473 & 35 & Ecopetrol & $\begin{array}{c}\text { ECOPET- } \\
\text { ROL }\end{array}$ & 0.177 & 0.461 & 0.638 \\
\hline 36 & $\begin{array}{l}\text { Resource } \\
\text { Generation }\end{array}$ & RES & 2.441 & 36 & $\begin{array}{l}\text { Imperial } \\
\text { Oil }\end{array}$ & IMO & 0.026 & 0.588 & 0.614 \\
\hline 37 & $\begin{array}{l}\text { Tata } \\
\text { Steel }\end{array}$ & TATASTEEL & 2.435 & 37 & BASF & BAS & 0.392 & 0.202 & 0.594 \\
\hline 38 & $\begin{array}{l}\text { Jastrzębska } \\
\text { Spółka } \\
\text { Węglowa }\end{array}$ & JSW & 2.355 & 38 & $\begin{array}{l}\text { BHP } \\
\text { Billiton }\end{array}$ & BHP & 0.312 & 0.227 & 0.539 \\
\hline 39 & $\begin{array}{l}\text { Adaro } \\
\text { Energy }\end{array}$ & ADRO & 2.242 & 39 & $\begin{array}{l}\text { Marathon } \\
\text { Oil }\end{array}$ & MRO & 0.129 & 0.410 & 0.539 \\
\hline 40 & $\begin{array}{l}\text { Shanxi } \\
\text { Lanhua } \\
\text { Sci-Tech }\end{array}$ & 600123 & 2.220 & 40 & $\begin{array}{c}\text { Cabot } \\
\text { Oil \& } \\
\text { Gas }\end{array}$ & COG & 0.510 & 0.025 & 0.535 \\
\hline 41 & $\begin{array}{l}\text { United } \\
\text { RUSAL }\end{array}$ & 0486 & 2.184 & 41 & $\begin{array}{l}\text { Anadarko } \\
\text { Petroleum }\end{array}$ & APC & 0.176 & 0.352 & 0.528 \\
\hline 42 & $\begin{array}{c}\text { AGL } \\
\text { Energy }\end{array}$ & AGK & 2.144 & 42 & $\begin{array}{l}\text { Conti- } \\
\text { nental } \\
\text { Resources }\end{array}$ & CLR & 0.226 & 0.271 & 0.497 \\
\hline 43 & $\begin{array}{c}\text { Rio } \\
\text { Tinto }\end{array}$ & RIO & 2.069 & 43 & Apache & APA & 0.127 & 0.312 & 0.439 \\
\hline 44 & $\begin{array}{c}\text { Shanghai } \\
\text { Datun } \\
\text { Energy }\end{array}$ & 600508 & 2.056 & 44 & Hess & HES & 0.106 & 0.327 & 0.433 \\
\hline 45 & Mitsui & 8031 & 1.956 & 45 & OMV & $\mathrm{OMV}$ & 0.181 & 0.242 & 0.422 \\
\hline 46 & $\begin{array}{c}\text { Kuz- } \\
\text { basskaya } \\
\text { Toplivna- } \\
\text { ya }\end{array}$ & KBTK & 1.890 & 46 & $\begin{array}{c}\text { CNX } \\
\text { Resources }\end{array}$ & CNX & 0.388 & 0.021 & 0.409 \\
\hline 47 & $\begin{array}{c}\text { Cloud } \\
\text { Peak } \\
\text { Energy }\end{array}$ & CLD & 1.886 & 47 & $\begin{array}{c}\text { Pioneer } \\
\text { Natural } \\
\text { Resource }\end{array}$ & PXD & 0.095 & 0.267 & 0.362 \\
\hline 48 & $\begin{array}{l}\text { CONSOL } \\
\text { Coal } \\
\text { Resources }\end{array}$ & CCR & 1.773 & 48 & $\begin{array}{l}\text { KazMun- } \\
\text { aiGas EP }\end{array}$ & RDGZ & 0.027 & 0.319 & 0.346 \\
\hline 49 & $\begin{array}{l}\text { New } \\
\text { Hope }\end{array}$ & NHC & 1.772 & 49 & YPF & YPFD & 0.137 & 0.190 & 0.327 \\
\hline 50 & $\begin{array}{l}\text { NLC } \\
\text { India }\end{array}$ & NLCINDIA & 1.591 & 50 & $\begin{array}{l}\text { Concho } \\
\text { Resources }\end{array}$ & $\mathrm{CXO}$ & 0.111 & 0.212 & 0.323 \\
\hline
\end{tabular}




\begin{tabular}{|c|c|c|c|c|c|c|c|c|c|}
\hline \multicolumn{4}{|c|}{$\begin{array}{l}\text { COAL RESERVES } \\
\text { (Top } 100 \text { Firms) }\end{array}$} & \multicolumn{6}{|c|}{$\begin{array}{c}\text { OIL \& GAS RESERVES } \\
\text { (Top } 100 \text { Firms) }\end{array}$} \\
\hline & \multirow{2}{*}{ Firms } & \multirow{2}{*}{ Ticker } & \multirow{2}{*}{$\mathrm{Gt} \mathrm{CO} \mathrm{CO}_{2}$} & & \multirow{2}{*}{ Firms } & \multirow{2}{*}{ Ticker } & Oil & Gas & $O \& G$ \\
\hline & & & & & & & $\mathrm{GtCO}$ & $\mathrm{Gt} \mathrm{CO}$ & Gt $\mathrm{CO}_{2}$ \\
\hline 51 & South32 & S32 & 1.586 & 51 & $\begin{array}{c}\text { Tourmaline } \\
\text { Oil }\end{array}$ & TOU & 0.264 & 0.055 & 0.320 \\
\hline 52 & Banpu & BANPU & 1.491 & 52 & $\begin{array}{l}\text { Woodside } \\
\text { Petroleum }\end{array}$ & WPL & 0.272 & 0.046 & 0.318 \\
\hline 53 & $\begin{array}{l}\text { NACCO } \\
\text { Industries }\end{array}$ & $\mathrm{NC}$ & 1.459 & 53 & Encana & ECA & 0.155 & 0.141 & 0.297 \\
\hline 54 & $\begin{array}{l}\text { Kangaroo } \\
\text { Resources }\end{array}$ & KRL & 1.434 & 54 & $\begin{array}{l}\text { Gulfport } \\
\text { Energy }\end{array}$ & GPOR & 0.263 & 0.031 & 0.294 \\
\hline 55 & Sumitomo & 80530 & 1.400 & 55 & Aker BP & AKERBP & 0.000 & 0.293 & 0.293 \\
\hline 56 & $\begin{array}{l}\text { Huolinhe } \\
\text { Coal }\end{array}$ & 002128 & 1.387 & 56 & $\begin{array}{l}\text { Husky } \\
\text { Energy }\end{array}$ & HSE & 0.087 & 0.191 & 0.279 \\
\hline 57 & $\begin{array}{c}\text { Golden } \\
\text { Energy } \\
\text { Mines }\end{array}$ & GEMS & 1.331 & 57 & $\begin{array}{c}\text { SK } \\
\text { Innovation }\end{array}$ & 096770 & 0.000 & 0.263 & 0.263 \\
\hline 58 & $\begin{array}{l}\text { Indika Inti } \\
\text { Corpindo }\end{array}$ & INDY & 1.182 & 58 & $\begin{array}{l}\text { Seven } \\
\text { Generations } \\
\text { Energy }\end{array}$ & VII & 0.126 & 0.134 & 0.260 \\
\hline 59 & $\begin{array}{c}\text { MC } \\
\text { Mining }\end{array}$ & MCM & 1.166 & 59 & $\begin{array}{c}\text { Murphy } \\
\text { Oil }\end{array}$ & MUR & 0.105 & 0.154 & 0.259 \\
\hline 60 & $\begin{array}{l}\text { Australian } \\
\text { Pacific } \\
\text { Coal }\end{array}$ & AQC & 1.147 & 60 & $\begin{array}{c}\text { QEP } \\
\text { Resources }\end{array}$ & QEP & 0.098 & 0.151 & 0.249 \\
\hline 61 & $\begin{array}{l}\text { Datang } \\
\text { Int. Power } \\
\text { Generation }\end{array}$ & 0991 & 1.147 & 61 & $\begin{array}{c}\text { Newfield } \\
\text { Exploration }\end{array}$ & NFX & 0.093 & 0.148 & 0.241 \\
\hline 62 & $\begin{array}{c}\text { Nippon } \\
\text { Steel \& } \\
\text { Sumitomo } \\
\text { Metal }\end{array}$ & 54010 & 1.023 & 62 & $\begin{array}{l}\text { California } \\
\text { Resources }\end{array}$ & CRC & 0.038 & 0.200 & 0.238 \\
\hline 63 & $\begin{array}{l}\text { Mongolian } \\
\text { Mining }\end{array}$ & 0975 & 0.999 & 63 & $\begin{array}{l}\text { Whiting } \\
\text { Petroleum }\end{array}$ & WLL & 0.046 & 0.188 & 0.234 \\
\hline 64 & Severstal & RTS2 & 0.869 & 64 & PTT & PTT & 0.166 & 0.066 & 0.232 \\
\hline 65 & $\begin{array}{c}\text { Beijing } \\
\text { Jingneng } \\
\text { Thermal } \\
\text { Powe }\end{array}$ & 600578 & 0.799 & 65 & Sasol & SOL & 0.069 & 0.156 & 0.225 \\
\hline 66 & $\begin{array}{l}\text { Shanxi } \\
\text { Meijin } \\
\text { Energy }\end{array}$ & 000723 & 0.784 & 66 & $\begin{array}{c}\text { Crescent } \\
\text { Point } \\
\text { Energy }\end{array}$ & CPG & 0.020 & 0.204 & 0.224 \\
\hline 67 & $\begin{array}{c}\text { Bayan } \\
\text { Resources }\end{array}$ & BYAN & 0.762 & 67 & $\begin{array}{c}\text { Birchcliff } \\
\text { Energy }\end{array}$ & BIR & 0.170 & 0.022 & 0.193 \\
\hline
\end{tabular}




\begin{tabular}{|c|c|c|c|c|c|c|c|c|c|}
\hline \multicolumn{4}{|c|}{$\begin{array}{l}\text { COAL RESERVES } \\
\text { (Top } 100 \text { Firms) }\end{array}$} & \multicolumn{6}{|c|}{$\begin{array}{c}\text { OIL \& GAS RESERVES } \\
\text { (Top } 100 \text { Firms) }\end{array}$} \\
\hline & \multirow{2}{*}{ Firms } & \multirow{2}{*}{ Ticker } & \multirow{2}{*}{$\mathrm{Gt} \mathrm{CO}{ }_{2}$} & & \multirow{2}{*}{ Firms } & \multirow{2}{*}{ Ticker } & Oil & Gas & O\&G \\
\hline & & & & & & & $\mathrm{GtCO}$ & $\mathrm{GtCO}$ & Gt CO \\
\hline 68 & $\begin{array}{l}\text { Jizhong } \\
\text { Energy }\end{array}$ & 000937 & 0.742 & 68 & $\begin{array}{c}\text { Cimarex } \\
\text { Energy }\end{array}$ & XEC & 0.088 & 0.103 & 0.191 \\
\hline 69 & $\begin{array}{l}\text { African } \\
\text { Rainbow } \\
\text { Minerals } \\
\text { Ltd }\end{array}$ & ARI & 0.742 & 69 & $\begin{array}{l}\text { Oil } \\
\text { India }\end{array}$ & OIL & 0.049 & 0.141 & 0.190 \\
\hline 70 & Allete & ALE & 0.723 & 70 & $\begin{array}{l}\text { WildHorse } \\
\text { Resource } \\
\text { Dev }\end{array}$ & WRD & 0.037 & 0.136 & 0.173 \\
\hline 71 & Wesfarmers & WES & 0.712 & 71 & $\begin{array}{c}\text { Ultra- } \\
\text { Petroleum }\end{array}$ & UPL & 0.161 & 0.012 & 0.173 \\
\hline 72 & Marubeni & 8002 & 0.706 & 72 & $\begin{array}{c}\text { MEG } \\
\text { Energy }\end{array}$ & MEG & 0.000 & 0.172 & 0.172 \\
\hline 73 & $\begin{array}{c}\text { Electric } \\
\text { Power } \\
\text { Develop- } \\
\text { ment }\end{array}$ & 9513 & 0.705 & 73 & Mitsui & 8031 & 0.107 & 0.063 & 0.170 \\
\hline 74 & $\begin{array}{c}\text { Idemitsu } \\
\text { Australia } \\
\text { Res }\end{array}$ & 5019 & 0.702 & 74 & Lundin & LUPE & 0.006 & 0.164 & 0.169 \\
\hline 75 & $\begin{array}{l}\text { Aspire } \\
\text { Mining }\end{array}$ & AKM & 0.670 & 75 & Energen & EGN & 0.031 & 0.135 & 0.166 \\
\hline 76 & $\begin{array}{l}\text { White } \\
\text { Energy }\end{array}$ & WEC & 0.653 & 76 & $\begin{array}{l}\text { WPX } \\
\text { Energy }\end{array}$ & WPX & 0.032 & 0.134 & 0.166 \\
\hline 77 & $\begin{array}{c}\text { Nava } \\
\text { Bharat } \\
\text { Ventures }\end{array}$ & 513023 & 0.612 & 77 & $\begin{array}{c}\text { SM } \\
\text { Energy } \\
\text { Comp }\end{array}$ & SM & 0.070 & 0.091 & 0.161 \\
\hline 78 & $\begin{array}{c}\text { Open } \\
\text { Joint Stock } \\
\text { Novolipetsk } \\
\text { Steel }\end{array}$ & NLMK & 0.606 & 78 & $\begin{array}{l}\text { PDC } \\
\text { Energy }\end{array}$ & PDCE & 0.063 & 0.097 & 0.159 \\
\hline 79 & $\begin{array}{l}\text { Hallador } \\
\text { Energy }\end{array}$ & HNRG & 0.599 & 79 & $\begin{array}{l}\text { Parsley } \\
\text { Energy }\end{array}$ & $\mathrm{PE}$ & 0.025 & 0.133 & 0.158 \\
\hline 80 & $\begin{array}{l}\text { Ramaco } \\
\text { Resources }\end{array}$ & METC & 0.573 & 80 & $\begin{array}{c}\text { Polish } \\
\text { Oil \& } \\
\text { Gas }\end{array}$ & PGN & 0.115 & 0.038 & 0.153 \\
\hline 81 & $\begin{array}{l}\text { Lubelski } \\
\text { Węgiel } \\
\text { Bogdanka }\end{array}$ & LWB & 0.572 & 81 & $\begin{array}{l}\text { Galp } \\
\text { Energia }\end{array}$ & GALP & 0.032 & 0.120 & 0.153 \\
\hline 82 & ArcelorMittal & MTL & 0.565 & 82 & $\begin{array}{c}\text { ARC } \\
\text { Resources }\end{array}$ & ARX & 0.107 & 0.045 & 0.152 \\
\hline
\end{tabular}




\begin{tabular}{|c|c|c|c|c|c|c|c|c|c|}
\hline \multicolumn{4}{|c|}{$\begin{array}{c}\text { COAL RESERVES } \\
\text { (Top } 100 \text { Firms) }\end{array}$} & \multicolumn{6}{|c|}{$\begin{array}{l}\text { OIL \& GAS RESERVES } \\
\text { (Top } 100 \text { Firms) }\end{array}$} \\
\hline & \multirow{2}{*}{ Firms } & \multirow{2}{*}{ Ticker } & \multirow{2}{*}{ Gt CO${ }_{2}$} & & \multirow{2}{*}{ Firms } & \multirow{2}{*}{ Ticker } & Oil & Gas & O\&G \\
\hline & & & & & & & $\mathrm{Gt} \mathrm{CO}_{2}$ & $\mathrm{Gt} \mathrm{CO}{ }_{2}$ & Gt $\mathrm{CO}_{2}$ \\
\hline 83 & $\begin{array}{l}\text { Up Energy } \\
\text { Development }\end{array}$ & 0307 & 0.554 & 83 & $\begin{array}{l}\text { Linn } \\
\text { Energy }\end{array}$ & LNGG & 0.094 & 0.054 & 0.148 \\
\hline 84 & $\begin{array}{c}\text { CLP } \\
\text { Holdings }\end{array}$ & 0002 & 0.552 & 84 & $\begin{array}{l}\text { Painted } \\
\text { Pony } \\
\text { Energy }\end{array}$ & PONY & 0.139 & 0.008 & 0.146 \\
\hline 85 & $\begin{array}{c}\text { Steel } \\
\text { Authority } \\
\text { of India }\end{array}$ & SAIL & 0.515 & 85 & $\begin{array}{c}\text { RSP } \\
\text { Permian }\end{array}$ & RSPP & 0.016 & 0.129 & 0.146 \\
\hline 86 & Vedanta & VEDL & 0.515 & 86 & $\begin{array}{c}\text { EP } \\
\text { Energy }\end{array}$ & $\mathrm{EPE}$ & 0.036 & 0.110 & 0.145 \\
\hline 87 & $\begin{array}{l}\text { Hindalco } \\
\text { Industries }\end{array}$ & 500440 & 0.512 & 87 & Santos & STO & 0.128 & 0.016 & 0.144 \\
\hline 88 & $\begin{array}{l}\text { LG } \\
\text { Corp }\end{array}$ & 003550 & 0.501 & 88 & $\begin{array}{l}\text { Peyto } \\
\text { E\&D }\end{array}$ & PEY & 0.123 & 0.010 & 0.133 \\
\hline 89 & $\begin{array}{l}\text { National } \\
\text { Aluminium }\end{array}$ & 532234 & 0.488 & 89 & $\begin{array}{c}\text { Oil } \\
\text { Search }\end{array}$ & OSH & 0.111 & 0.021 & 0.132 \\
\hline 90 & Sojitz & 2768 & 0.485 & 90 & $\begin{array}{l}\text { Dia- } \\
\text { mond- } \\
\text { back } \\
\text { Energy }\end{array}$ & FANG & 0.016 & 0.115 & 0.131 \\
\hline 91 & $\begin{array}{l}\text { Agritrade } \\
\text { Resources }\end{array}$ & 1131 & 0.482 & 91 & ENGIE & ENGI & 0.096 & 0.034 & 0.130 \\
\hline 92 & $\begin{array}{l}\text { Rhino } \\
\text { Resource } \\
\text { Partners }\end{array}$ & RHNOD & 0.478 & 92 & $\begin{array}{l}\text { JXTG } \\
\text { Holdings }\end{array}$ & 5020 & 0.068 & 0.058 & 0.126 \\
\hline 93 & FirstEnergy & $\mathrm{FE}$ & 0.463 & 93 & $\begin{array}{c}\text { Oasis } \\
\text { Petroleum }\end{array}$ & OAS & 0.029 & 0.095 & 0.124 \\
\hline 94 & $\begin{array}{l}\text { Kinetic } \\
\text { Mines } \\
\text { and } \\
\text { Energy }\end{array}$ & 1277 & 0.448 & 94 & $\begin{array}{l}\text { Sanchez } \\
\text { Energy }\end{array}$ & SN & 0.042 & 0.081 & 0.123 \\
\hline 95 & PGE & PGE & 0.436 & 95 & $\begin{array}{l}\text { National } \\
\text { Fuel Gas }\end{array}$ & NFG & 0.108 & 0.013 & 0.120 \\
\hline 96 & $\begin{array}{l}\text { JXTG } \\
\text { Hldgs }\end{array}$ & 5020 & 0.428 & 96 & $\begin{array}{l}\text { Whitecap } \\
\text { Resources }\end{array}$ & WCP & 0.015 & 0.104 & 0.119 \\
\hline 97 & $\begin{array}{l}\text { Prairie } \\
\text { Mining }\end{array}$ & PDZ & 0.428 & 97 & $\begin{array}{l}\text { Oando } \\
\text { PLC }\end{array}$ & OANDO & 0.064 & 0.051 & 0.114 \\
\hline 98 & $\begin{array}{c}\text { Feishang } \\
\text { Anthracite } \\
\text { Resources }\end{array}$ & 1738 & 0.413 & 98 & $\begin{array}{l}\text { Denbury } \\
\text { Resources }\end{array}$ & DNR & 0.002 & 0.107 & 0.109 \\
\hline
\end{tabular}




\begin{tabular}{|c|c|c|c|c|c|c|c|c|c|}
\hline \multicolumn{4}{|c|}{$\begin{array}{l}\text { COAL RESERVES } \\
\text { (Top } 100 \text { Firms) }\end{array}$} & \multicolumn{6}{|c|}{$\begin{array}{l}\text { OIL \& GAS RESERVES } \\
\text { (Top } 100 \text { Firms) }\end{array}$} \\
\hline & \multirow{2}{*}{ Firms } & \multirow{2}{*}{ Ticker } & \multirow{2}{*}{ Gt $\mathrm{CO}_{2}$} & \multirow{2}{*}{\multicolumn{2}{|c|}{ Firms }} & \multirow{2}{*}{ Ticker } & Oil & Gas & O\&G \\
\hline & & & & & & & Gt $\mathrm{CO}_{2}$ & Gt $\mathrm{CO}_{2}$ & Gt $\mathrm{CO}_{2}$ \\
\hline 99 & $\begin{array}{c}\text { Realm } \\
\text { Resources }\end{array}$ & RRP & 0.409 & 99 & $\begin{array}{l}\text { Paramount } \\
\text { Resources }\end{array}$ & POU & 0.071 & 0.036 & 0.107 \\
\hline 100 & $\begin{array}{l}\text { Shanxi } \\
\text { Coking }\end{array}$ & 600740 & 0.383 & 100 & $\begin{array}{l}\text { Extraction } \\
\text { Oil \& Gas }\end{array}$ & XOG & 0.034 & 0.072 & 0.106 \\
\hline \multicolumn{3}{|c|}{$\begin{array}{c}\text { Total Carbon } \\
\text { Gt } \mathrm{CO}_{2}\end{array}$} & 368.57 & \multicolumn{3}{|c|}{$\begin{array}{l}\text { Total Oil \& } \\
\text { Gas Gt } \mathrm{CO}_{2}\end{array}$} & 78.13 & 74.28 & 152.65 \\
\hline \multicolumn{7}{|c|}{ Total Carbon, Oil, \& Gas Asset Reserves } & \multicolumn{3}{|c|}{$520.988 \mathrm{GtCO}_{2}$} \\
\hline
\end{tabular}

Table 1: The Carbon Underground 200 (Fossil Free Indexes, 2018)

\section{Divestment in Higher Education}

The divestment movement in higher education has been grounded in exposing moral hypocrisy in universities that have been entrusted with preparing students for their future and yet seek at the same time to profit from an industry that is destroying it. Divestment advocates call on these higher education institutions (as well as on foundations, religious institutions, municipalities, and others) to halt new investments in fossil fuel companies and phase out existing investments within a few years. However, while the movement has spread that demand to over 500 campuses in the United States as of Spring 2019, relatively few institutions have formally committed to it (most, in fact, have formally rejected it).

According to Michelle Raji, the first student-led fossil free initiative started at Swarthmore College in 2011 (Raji, 2014). The movement did not gain substantial traction, however, until 2014, when McKibben's Fossil Free campaigns helped develop collective action by supporting global student movement in university campuses. During the first nine months of that year, the number of universities that pledged to divest from fossil fuel firms more than doubled from 74 to 181 (Grady-Benson \& Sarathy, 2016). In 2015, the University of California system sold $\$ 200$ million worth of endowment and pension fund holdings in coal and oil sands companies. Stanford University, in response to student protests, committed to divest from coal companies in 2014, and agreed in November 2015 to consider dropping investments in oil and gas from its $\$ 21$ billion endowment portfolio (Hiltzik, 2016). 
Universities still have a certain legitimacy that grants them the potential for leverage particularly in a landscape where many people have lost faith in the principal sectors of power. Divestment actions make news precisely because such movements are unusual and because they are based on moral grounds. In the grand scheme of things, however, total university endowment in the United States, valued at $\$ 650$ to $\$ 660$ billion, is many orders of magnitude smaller than the global energy industry, which is valued at $\$ 4$ to $\$ 5$ trillion. Estimates indicate, moreover, that only around $2 \%$ to $4 \%$ of U.S. university endowments, and around $5 \%$ in the United Kingdom, are invested in fossil fuel companies (Ansar et al., 2013; Bullard, 2014). Why bother, then? We cannot discover, of course, how much influence the legitimacy of universities will grant them if they do not attempt to use it, yet we might believe from historical precedent that such efforts may inspire and create change, even when they begin on a small scale (Gitlin, 2013). Sociologist, author, and long-time activist Todd Gitlin wrote:

Those in the growing divestment movement suffer no illusions that universities themselves wield the magnitude of power you find in investment banks or, of course, the FFCs [Fortune 500 companies] themselves. They are simply seeking leverage where they can. (2013)

When the Rockefeller Brothers Fund, a philanthropy whose funds originally came from oil and gas, announced that it would sell off its holdings in fossil fuel companies, the fund's president, Stephen Heintz, acknowledged the divestment as being almost purely symbolic, with relatively little impact on either shareholders or institutions. He maintained, however, that symbols are important: " "This (action) is largely symbolic, but symbols have power. They motivate people. They inspire people. They can change behavior" (Stephen Heintz, quoted in Gunther, 2015).

\section{Fossil Fuel Divestment Initiatives in U.S.-based Jesuit Universities}

Students have been the ones leading the divestment movement in Jesuit universities. Various groups have spearheaded divestment initiatives, including Climate Justice at Boston College, ${ }^{7}$ Boston College Alumni for Divestment, Creighton University Climate Movement, Holy Cross Fossil Free, Fossil Free Fordham, Fossil Free Georgetown University, Fossil Free Gonzaga, Divest Gonzaga, Student Environmental

${ }^{6} \mathrm{~A}$ list of all firms and institutions that have divested from fossil fuel firms is available at https:// gofossilfree.org/divestment/commitments/.

${ }^{7}$ Students voted for and approved a resolution demanding FFD in 2019. 
Alliance at Loyola University Chicago, ECO Students Loyola Marymount University (LMU), Fossil Free LMU, Divest LMU, Fossil Free Loyola University New Orleans, Divest Loyola New Orleans, and Divest Santa Clara University, among others. The Divestment Jesuit Network, also led by students, has created an organized consortium of Jesuit universities that collectively shares resources and strategies; participating schools are College of the Holy Cross, Loyola University New Orleans, University of Scranton, Loyola Marymount University, Boston College, Georgetown University, Loyola University Chicago, Canisius College, and Santa Clara University.

Many, or maybe even most, faculty members in Jesuit universities also support divestment. They argue that universities, as institutions of research and learning, must acknowledge and respond to the science they teach, a science that shows how continued reliance on fossil fuels contributes to a changing climate and global warming. Faculty, however, albeit with a few exceptions, ${ }^{8}$ have preferred to remain in the background and let students take the lead.

\section{METHODOLOGY AND DATA COLLECTION}

Data on FFD was obtained through the public database of 350.org (Fossil Free, n.d.), the website led by environmental activist Bill McKibben who adopted the FFD cause by embarking on a bus tour across the U.S. to promote divestment. Endowment data was obtained through the public database of the National Association of College and University Business Officers, ${ }^{9}$ an association of the higher education industry that provides a training, networking, and advocacy platform for financial executives. To evaluate divestment initiatives in U.S.-based Jesuit universities, data on the universe of comparable private 4-year universities in the U.S. was obtained through the Institutional Characteristics component of the Integrated Postsecondary Education Data System (IPEDS) run by the U.S. Department of Education's National Center for Education Statistics. IPEDS data was also used to establish a baseline for comparison.

${ }^{8}$ In early 2015, 202 faculty members at Loyola University in Chicago called for divestment in a six-page letter to the Faculty Senate, which passed a resolution in their favor. This led to amendments in university investment policy which included Responsible and Sustainable Investing Principles (https://www.intentionalendowments.org/loyola_university_chicago).

${ }^{9}$ Cross-referenced with IPEDS. Endowment and divestment status data were sent to all U.S.based Jesuit universities for verification. 
The websites of the 27 Jesuit universities in the U.S. were examined to investigate their 1) general environmental/sustainability initiatives, 2) endowment investment policy and data (if available), and 3) if they had a formal position on FFD. A general Internet and news search was also conducted to examine all FFD-related news and check if there were any FFD advocacy student groups affiliated with these universities.

The Office of the President, business school deans, and financial officers of each U.S.-based Jesuit university were contacted by email during the Spring and Summer of 2019 to verify 1) endowment data, 2) divestment status, and 3) information regarding any pending FFD initiatives. Information about the universities' policies regarding investments in fossil fuel firms was also requested. ${ }^{10}$ Emails were resent twice for non-respondents early in the Fall of 2019. The Offices of the President for most of the universities redirected our requests to financial officers who were more actively involved in the divestment process. These officers responded by email; some agreed to telephone interviews. The study also maintained confidentiality for all respondents to avoid a breach by process of elimination (given the small number of respondents in total), even though some did not request it.

The universe of all private 4-year universities in the United States was crosschecked to obtain a sub-sample of all those that subscribed to FFD. Public universities were excluded since U.S.-based Jesuit universities are private institutions. University data on divestment was also adjusted accordingly given that IPEDS accounts for branch campuses as separate institutions.

\section{RESULTS}

Of the 27 Jesuit universities that were contacted, 13 responded (48.15\%) and confirmed the accuracy of our data. Ten of them also provided various degrees of information related to their 1) evaluation process for rejecting and/or approving FFD (e.g., administrative, Board of Trustees vote, etc.); 2) reasons for deciding for or against FFD; and 3) exposure to or percentage of investments in fossil fuel firms.

The responses obtained indicate that only two U.S.-based Jesuit universities so far have affirmatively committed to a fossil fuel-free investment portfolio: Georgetown

${ }^{10} \mathrm{~A}$ list of which firms are considered "fossil fuel firms" was also sent to all universities. See Table 1 
University and Seattle University. Nevertheless, results show that after controlling for branch campuses, $13.33 \%$ of all Jesuit universities had agreed to FFD compared to only $4.12 \%$ of all 4 -year private universities in the U.S. ${ }^{11}$

\begin{tabular}{|c|c|c|}
\hline \multicolumn{3}{|c|}{$\begin{array}{c}\text { ENDOWMENTS AND FOSSIL FUEL DIVESTMENT IN } \\
\text { U.S.-BASED JESUIT UNIVERSITIES }\end{array}$} \\
\hline UNIVERSITY & $\begin{array}{l}\text { ENDOWMENT } \\
(\text { AS OF 2019)* }\end{array}$ & DIVESTMENT STATUS** \\
\hline Boston College & $\$ 2,523,300,000$ & rejected FFD \\
\hline Canisius College $^{[1]}$ & $\$ 133,900,000$ & rejected FFD \\
\hline College of the Holy Cross & $\$ 785,852,000$ & not divested \\
\hline Creighton University & $\$ 587,024,341$ & rejected $\mathrm{FFD}^{[5]}$ \\
\hline Fairfield University & $\$ 374,900,000$ & not divested \\
\hline Fordham University & $\$ 733,516,000$ & rejected FFD \\
\hline Georgetown University & $\$ 1,822,484,000$ & fully divested \\
\hline Gonzaga University & $\$ 294,720,414$ & rejected FFD \\
\hline John Carroll University & $\$ 223,538,691$ & not divested \\
\hline Le Moyne College & $\$ 187,600,000$ & rejected FFD \\
\hline Loyola College (Maryland) & $\$ 232,472,905$ & not divested \\
\hline Loyola Marymount University & $\$ 477,600,000$ & not divested \\
\hline Loyola University Chicago ${ }^{[2]}$ & $\$ 692,166,000$ & not divested \\
\hline Loyola University (New Orleans) & $\$ 235,500,000$ & not divested \\
\hline Marquette University & $\$ 698,021,348$ & not divested \\
\hline Regis University & $\$ 69,772,662$ & not divested \\
\hline Rockhurst College ${ }^{[3]}$ & $\$ 37,100,000$ & not divested, pending \\
\hline Saint Joseph's University & $\$ 294,286,969$ & not divested \\
\hline Saint Louis University & $\$ 1,252,677,869$ & not divested \\
\hline Saint Peter's College & $\$ 31,050,000$ & not divested \\
\hline Santa Clara University & $\$ 1,019,760,000$ & rejected FFD \\
\hline Seattle University & $\$ 241,200,000$ & fully divested \\
\hline
\end{tabular}

${ }^{11} \mathrm{~A}$ total of 65 private 4-year universities in the U.S. agreed to some type of FFD as of April 30, 2020. There were a total of 1,577 4-year universities in the U.S. as of June 2019 (IPEDS, 2019; branch campuses are accounted for as separate institutions). For comparison with the IPEDS data, we adjusted the FFD data obtained from 350.org to account for branch campuses as separate institutions. The percentage of FFD commitments from public 4-year universities is similar at $4.156 \%$. 


\begin{tabular}{|c|c|c|}
\hline \multicolumn{3}{|c|}{$\begin{array}{c}\text { ENDOWMENTS AND FOSSIL FUEL DIVESTMENT IN } \\
\text { U.S.-BASED JESUIT UNIVERSITIES }\end{array}$} \\
\hline UNIVERSITY & $\begin{array}{c}\text { ENDOWMENT } \\
(\text { AS OF 2019) }\end{array}$ & DIVESTMENT STATUS $^{* *}$ \\
\hline Spring Hill College & $\$ 24,767,781$ & not divested \\
\hline University of Detroit Mercy & $\$ 69,300,000$ & not divested \\
\hline University of San Francisco & $\$ 399,571,000$ & not divested \\
\hline University of Scranton & $\$ 209,760,436$ & not divested \\
\hline Wheeling Jesuit College & $\$ 16,100,000$ & not divested \\
\hline Xavier University ${ }^{[4]}$ & $\$ 199,275,000$ & not divested \\
\hline TOTAL ENDOWMENTS & \multicolumn{2}{|c|}{$\$ 13,867,217,416$} \\
\hline
\end{tabular}

Table 2: Endowments and Fossil Fuel Divestment in U.S.-based Jesuit Universities

*Endowment market value as of June 2019 (National Association of College and University Business Officers [NACUBO])

${ }^{\star \star}$ As of July 2019 (Fossil Free, n.d., and as disclosed by university officials)

"Rejected FFD" indicates an explicit decision not to divest from fossil fuels by the formal or informal statement or vote of the administration, President, or Board of Trustees.

Estimated fossil fuel exposure, \% of invested assets, as disclosed by university officials: $[1]=1 \% ;[2]=2 \% ;[3]=1.72 \% ;[4]=1.1 \%$; [5] reduced to $5.76 \%$

Table 2 shows the endowments of U.S.-based Jesuit universities as of June 2019 along with their divestment status and, in some cases, the voluntary disclosure of their percentage exposure in the stocks of fossil fuel firms (see Table 2 notes). The total endowments for all 27 U.S.-based Jesuit universities add up to \$13.86 billion. Of the two Jesuit universities that have committed to full divestment from all fossil fuel firms, defined as those among the 200 largest in terms of oil, gas, and carbon reserves (Fossil Free Indexes, 2018), the first to commit was Seattle University (in September of 2018). Georgetown, on the other hand, was one of the early adopters when it agreed in 2015 to "divest partially" from firms associated with coal and tar sands extraction. Their Board of Directors eventually committed to full divestment in early February of 2020.

It is worth mentioning that Campion College-Regina, a Jesuit institution in Canada, agreed to divest from all fossil fuel firms in 2016 and is now fossil fuel-free. There is also evidence that other Jesuit universities outside the United States, like ESADE in Barcelona, Spain, have also divested and are now fossil fuel-free. 
Our email and telephone interviews provided opportunities to explore the reasons for not adopting an FFD strategy; these have not been attributed to any specific university as many of these responses were given in confidentiality. Arguments related to finances made up the overwhelmingly predominant reason for rejecting FFD, providing evidence that the decision against divesting appears to be financial in nature and the product of a cost-benefit analysis.

\section{Why Universities Agree to Divest}

As reported by G. Jeffrey MacDonald (2019), universities committed to fossil fuel divestment argue that their existing mission made divestment a process of aligning proposed action with existing cultural values. These divestment adopters leaned on the strength of their cultural values to overcome a powerful market logic that demanded financial growth from endowments. They were also highly active in climate change issues before considering divestment, and were already engaged in most instances in the socially responsible management of their endowment portfolios such that fossil fuel divestment easily fit into their existing institutional logic and aligned with their prioritized cultural values, vision, and mission. Analysis of the responses in this study also revealed that the alignment of cultures of sustainability, often expressed explicitly in the universities' mission statements, was a key rationale for committing to divestment. Many of the divesting universities framed their investment decisions as necessary for aligning what they were teaching in the classrooms with their administrative actions. Lastly, rather than fully rejecting the fiscal growth imperative, many cited a belief that fossil fuel-free portfolios would perform as well or better than those that did not divest. They actively engaged in developing a socially responsible investment strategy that placed boundaries around what was acceptable without rejecting financial rationale. Others also shared similar stories of having aligned their investment strategies with social and environmental concerns even before the FFD campaign emerged. 
As a Jesuit and Catholic university we have a special obligation to address the unfolding climate change crisis. In his encyclical Laudato Si', or Care for Our Common Home, Pope Francis calls us to view this as a social and ecological issue of grave urgency that is connected to all around us and that has especially devastating consequences for society's most vulnerable.

—Stephen V. Sundborg, S.J. President, Seattle University $(\text { September 19, 2018) })^{12}$

[Pope Francis's] words inform and strengthen our commitment to the environment, and to one another... The work of understanding and responding to the demands of climate change is urgent and complex.

\section{REFLECTIONS}

Divestment from fossil fuels is a way for all educational institutions-Jesuit, Roman Catholic, or otherwise-to live the challenge issued by Francis in Laudato Si'. Describing environmental stewardship as a moral obligation for all humanity, the 2015 papal encyclical adds to the discussions of more faith communities as they look in particular at their investment policies. It sets up a moral tone that has led many religious denominations to divest from at least some activities linked to fossil fuels. Such acts of divestment by religious institutions call ordinary citizens to consider the morality of profiting from fossil fuel-related businesses, and remind broad audiences that while some concrete activities contribute to creating climate change, others can contribute to dealing with it. Indeed, our collective failure to respond to the crisis, highlighted by the current evidence of rising temperatures, points, as Francis states, "to the loss of that sense of responsibility for our fellow men and women upon which all civil society is founded" (Francis, 2015: no. 25).

${ }^{12}$ Available at https://www.seattleu.edu/president/update/seattle-university-board-votes-todivest-from-fossil-fuels.html. 
What does this exploration say, then, about the future of the fossil fuel divestment campaign? The explanations of adopters indicate that we can safely expect other Jesuit campuses, which also prioritize environmental sustainability as a core value of their identity, to be primed for adopting the demands of the divestment campaign. The values of sustainability, in particular, will have to be more resilient, albeit without fully replacing the institutional logic of the market. We expect that colleges that divest in the future will frame their decision according to the values of their institutional mission but couched in a language familiar to the logics of finance-growth, risk, market signals, and fiduciary responsibility. Individual campaigns may also find success when they can frame the issue of divestment around the local cultural values of each campus without straying too far from market concerns. The scope of the climate change crisis, however, ultimately still requires collective action.

This and the next paper in this issue of the Journal advocate for all universities, and Roman Catholic and Jesuit institutions in particular, to join the Fossil Fuel Divestment movement and sell (divest) whatever financial holdings they might have in fossil fuel firms. Jesuit universities have an opportunity both to provide global leadership by acting according to their missions and underlying mandate of social justice and to build in general on the moral leadership Francis has offered in his courageous attempt to stop this insanity.

The campaigns demanding FFD that have been mounted by students from Jesuit universities are particularly impressive in that they have articulated a message that goes beyond the impact of fossil fuels on climate change. Students have explicitly argued in favor of FFD as a moral imperative expressed by Francis in Laudato Si' and as a testimony of their own faith. They have also formed impressive alliances across all Jesuit universities, providing a remarkable model of how Jesuit institutions can leverage a common identity and articulating in some way that the scope of the climate change crisis requires collective action. After all, it may be that these student movements are, regardless of the outcome, a demonstration of their Jesuit formation, and we should be proud of that. As for faculty, they have not been heavily involved in the FFD movement, though there is anecdotal evidence that they do support it. There have also been sporadic 
initiatives in faculty senates and assemblies in support of FFD; ${ }^{13}$ hopefully there will be more.

The next article, which explores the arguments for and against divestment that were identified through our communications with Jesuit universities, suggests that any university committed to creating a sustainable world can "walk the talk" of their espoused commitments and values of sustainability and environmental stewardship without having to suffer in terms of endowment portfolio returns. They can still honor their values and mission without having to pay a financial price for doing so, even if they take the very narrow and hard-to-defend position that their "fiduciary responsibilities" are restricted only to how much, and not to how, their portfolios earn.

Fossil fuel divestment in Jesuit universities is a moral imperative. It can be an opportunity to align actions with all the talk about sustainability and social justice. It is a chance not just to "talk the talk" but to "walk the walk."

\section{REFERENCES}

Ansar, A., Caldecott, B., \& Tilbury, J. 2013. Stranded assets and the fossil fuel divestment campaign: What does divestment mean for the valuation of fossil fuel assets? Stranded Assets Programme, Smith School of Enterprise and the Environment, University of Oxford. Available at https://www.smithschool. ox.ac.uk/publications/reports/SAP-divestment-report-final.pdf (accessed May 19, 2019).

Apfel, D. 2015. Exploring divestment as a strategy for change: An evaluation of the history, success, and challenges of fossil fuel divestment. Social Research, 82(4): 913-937.

Ayling, J., \& Gunningham, N. 2017. Non-state governance and climate policy: The fossil fuel divestment movement. Climate Policy, 17(2): 131-149.

${ }^{13}$ Faculties of Boston College, Canisius College, Gonzaga University, and Loyola University Chicago approved resolutions in support of FFD. 
Barca, J. 2018. Florida school shooting prompts Dick's Sporting Goods to end sale of assault weapons. Forbes, February 28. Available at https://www.forbes.com/sites/ jerrybarca/2018/02/28/florida-school-shooting-prompts-dicks-sporting-goods-toend-sale-of-assault-weapons/\#24fec0405cbb.

Barnett, M. 2007. Stakeholder influence capacity and the variability of financial returns to corporate social responsibility. Academy of Management Review, 32(3): 794-816.

Benford, R. D. 1993. "You could be the hundredth monkey": Collective action frames and vocabularies of motive within the nuclear disarmament movement. Sociological Quarterly, 34(2): 195-216.

Bullard, N. 2014. Fossil fuel divestment: A \$5 trillion challenge. Bloomberg New Energy Finance, August 25: 1-19.

Clark, M. 2011. Faithful take money out of big banks to protest corporate greed. National Catholic Reporter, October 14. Available at https://www.ncronline. org/news/faithful-take-money-out-big-banks-protest-corporate-greed.

Finn, S. P. 2019. Faith consistent investment guidelines. In L. Hogan, M. Becka, \& J. Vila-Chã (Eds.), Technology: Between apocalypse and integration, Concilium 2019/3: 131-135. London: SCM Press.

Fossil Free. n.d. 1000+ divestment commitments. Available at https://gofossilfree. org/divestment/commitments/.

Fossil Free Indexes. 2018. The carbon underground 200. Port Washington, NY: Fossil Free Indexes.

Francis. 2015. Laudato si': On care for our common home. Vatican City: Libreria Editrice Vaticana.

Freedman, A. 2020. This year is on track to be Earth's warmest on record, beating 2016, NOAA says. The Washington Post, April 21. Available at https://www. washingtonpost.com/weather/2020/04/21/earth-warmest-year-likely-2020/. 
Friedman, M. 1970. The social responsibility of business is to increase its profits. The New York Times Magazine, September 13.

Gillis, J. 2018. He was right about the climate. The New York Times, June 24: SR11ff.

Gitlin, T. 2013. How to reverse a slow-motion apocalypse: Why the divestment movement against Big Energy matters. TomDispatch.com, November 21.

Goodwin, P., Katavouta, A., Roussenov, V. M., Foster, G. L., Rohling, E. J., \& Williams, R. G. 2018. Pathways to $1.5^{\circ} \mathrm{C}$ and $2^{\circ} \mathrm{C}$ warming based on observational and geological constraints. Nature Geoscience, 11(2): 102-107.

Grady-Benson, J., \& Sarathy, B. 2016. Fossil fuel divestment in US higher education: Student-led organising for climate justice. Local Environment, 21(6): 661-681.

Gunningham, N. 2017. Building norms from the grassroots up: Divestment, expressive politics, and climate change. Law \& Policy, 39(4): 372-392.

Gunther, M. 2015. Why the fossil fuel divestment movement may ultimately win. Yale Environment 360, July 27. Available at https://e360.yale.edu/features/ why_the_fossil_fuel_divestment_movement_may_ultimately_win (accessed June 3, 2019).

Hawken, P. (Ed.). 2017. Drawdown: The most comprehensive plan ever proposed to reverse global warming. New York: Penguin.

Heede, R. 2014. Tracing anthropogenic carbon dioxide and methane emissions to fossil fuel and cement producers, 1854-2010. Climatic Change, 122(1-2): 229-241.

Hiltzik, M. 2016. When is it worth it to divest? Los Angeles Times, January 17: C1-C8.

Hodgson, J. 2009. Catholic principles guide mutual funds. The Wall Street Journal, 254(89): B6. 
IPCC [Intergovernmental Panel on Climate Change]. 2018. Summary for policymakers. In V. Masson-Delmotte, P. Zhai, H.-O. Pörtner, D. Roberts, J. Skea, P. R. Shukla, A. Pirani, W. Moufouma-Okia, C. Péan, R. Pidcock, S. Connors, J. B. R. Matthews, Y. Chen, X. Zhou, M. I. Gomis, E. Lonnoy, T. Maycock, M. Tignor, $\&$ T. Waterfield (Eds.), Global warming of $1.5^{\circ} \mathrm{C}$ : An IPCC special report on the impacts of global warming of $1.5^{\circ} \mathrm{C}$ above pre-industrial levels and related global greenhouse gas emission pathways, in the context of strengthening the global response to the threat of climate change, sustainable development, and efforts to eradicate poverty: 1-24. World Meteorological Organization, United Nations.

IPEDS [Integrated Postsecondary Education Data System]. 2019. IPEDS Survey components > Institutional characteristics. Integrated Postsecondary Education Data System. National Center for Education Statistics, U.S. Department of Education. Available at https://nces.ed.gov/ipeds/use-the-data/surveycomponents/4/institutional-characteristics.

Kimbro, M. B. 2018. Innovation for sustainability: A call for an abolitionist mindset to liberate our imagination for action. Journal of Management for Global Sustainability, 6(2): 1-17.

Kolbert, E. 2014. The sixth extinction: An unnatural history. London: Bloomsbury Publishing.

Krantz, M. 2015. "Pope portfolio" helps elevate investing. USA Today, September 28: Money, 1.

Linnenluecke, M. K., Meath, C., Rekker, S., Sidhu, B. K., \& Smith, T. 2015. Divestment from fossil fuel companies: Confluence between policy and strategic viewpoints. Australian Journal of Management, 40(3): 478-487.

Loder, A. 2017. Ethical funds are hard to define. The Wall Street Journal, May 5: B1-B5.

MacDonald, J. 2019. Where your treasure is (or isn't): The debate over shareholder activism and divestment. The Christian Century, January 2: 20-22. 
McKibben, B. 2013. The case for fossil-fuel divestment: On the road with the new generation of college activists fighting for the environment. Rolling Stone, February 22.

Muntean, M., Guizzardi, D., Schaaf, E., Crippa, M., Solazzo, E., Olivier, J. G. J., \& Vignati, E. 2018. Fossil $\mathrm{CO}_{2}$ emissions of all world countries - 2018 report. Luxembourg: Publications Office of the European Union.

Neuman, W. 2018. To fight climate change, New York City takes on oil companies. The New York Times, January 10.

Raji, M. Y. 2014. Timeline: Fossil fuels divestment. The Harvard Crimson, October 2. Available at https://www.thecrimson.com/article/2014/10/2/timeline-fossilfuels-divestment/ (accessed March 29, 2019).

Rich, N. 2018. Losing Earth: A recent history. New York: MCD.

Roewe, B. 2014. Scholars explore Catholic path to fossil fuel divestment. National Catholic Reporter, May 23-June 5: Nation, 17.

Roewe, B. 2016. As Paris Agreement enters into force, focus turns to making it work. National Catholic Reporter, October 18.

Seidman, G. 2015. Divestment dynamics: Mobilizing, sharing, and changing the rules. Social Research, 82(4): 1015-1037.

Shabecoff, P. 1988. Global warming has begun, expert tells Senate. The New York Times, June 24.

Svrluga, S. 2020. Georgetown University says it will divest from fossil fuel companies. The Washington Post, February 7.

Weis, W. L. 2013. Hypocrisy at the lectern: Do our personal lifestyle choices reflect our spoken commitment to global sustainability? Journal of Management for Global Sustainability, 1(1): 29-45.

WWF [World Wildlife Fund]. 2018. Living planet report 2018: Aiming higher. M. Grooten \& R. E. A. Almond (Eds.). Gland, Switzerland: WWF. 
Marinilka Barros Kimbro is an Associate Professor of Accounting at the Albers School of Business and Economics, Seattle University. She received a Ph.D. in Accounting from the University of Maryland, College Park and has held research appointments at the University of Washington, Groupe HEC-Paris, Hong Kong Polytechnic, IE-Madrid, and Gonzaga University. Her scholarship examines the role of information in firm risk, shareholders' activism, executive compensation, and decision making. Her publications appear in the Journal of Accounting and Public Policy, Journal of Accounting, Auditing \& Finance, Asia-Pacific Journal of Accounting \& Economics, and Journal of International Financial Management \& Accounting, among others. Her research attempts to generate "engaged scholarship" that is relevant to her teaching, to the profession, and that expresses Seattle University's mission of social justice.

Rubina Mahsud is a thought leader in business education, corporate strategy, and leadership whose work spans from humanitarian services to return on shareholder equity. Her articles have appeared in the Journal of Managerial Psychology, Journal of Leadership and Organizational Studies, Personnel Review, and Business and Society Review. She currently serves as an Associate Professor of Management at the Center for Leadership Formation, Albers School of Business \& Economics at Seattle University. Dr. Mahsud holds her Ph.D. in Management as well as a master's degree in Public Health from the State University of New York at Albany. Her prior degrees include a master's in Social Sciences from the University of Birmingham (U.K.) and a medical degree from Jinnah Medical College (Pakistan).

Davit Adut, Ph.D., is an Assistant Professor of Accounting at the Albers School of Business and Economics, Seattle University whose research focuses on the effect of accounting information on executive compensation, analysts' forecasts, and corporate governance. He has held research appointments at the American University and University of Cincinnati, and his publications appear in Accounting Review, Journal of Accounting and Public Policy, and Advances in Accounting. Dr. Adut also serves as the senior editor for International Accounting, Auditing, and Taxation. 